\title{
Los modos de vida y la paradoja de la ética contemporánea
}

M. Adrián Almazán Gómez Universidad Autónoma de Madrid (UAM) adrian.almazan@uam.es

\section{Introducción. La paradoja ética de nuestro tiempo}

El mundo de hoy se encuentra atravesado por una tremenda paradoja. Por un lado, en ningún otro momento la ética ha tenido una presencia y relevancia social mayores. La extensión de los comités de ética, las comisiones evaluadoras o el análisis de riesgo, la profunda especialización y división de la misma, a la par que la enorme preocupación social por la corrección política, dibujan un escenario de inflación de la ética que, a veces, puede llegar a generar la sensación de una presencia desorbitada y asfixiante de los imperativos éticos en la vida social (Barba, 2016).

Sin embargo, junto a esta hipertrofia convive una alarmante impotencia para evaluar y cambiar el curso de las transformaciones a las que se ven sujetos nuestros modos de vida. En los últimos siglos, pero con especial virulencia en las décadas que distan del fin de la Segunda Guerra 
Mundial, procesos como la globalización económica, la artificialización de la vida y de los procesos de producción y reproducción social, la tecnologización de las sociedades, la deriva securitaria, la polarización social, la crisis ecológica, etc., han trastocado los modos de vida en todo el mundo sin aparente posibilidad de enmienda por parte de las sociedades y las personas afectadas. Plantearse la que quizá es la pregunta más importante: ¿qué mundo es el que realmente queremos?, parece haberse tornado imposible.

A esta situación dual es a la que el profesor de filosofía moral de Lovaina, Mark Hunyadi (2015) se refiere como la paradoja ética de nuestro tiempo. Debido a que el resultado de dicha paradoja es precisamente una imposibilidad de la ética contemporánea -y de sus muchas subdisciplinas, como la bioética- para abordar problemas estructurales graves como la crisis socioeconómica que jalona los inicios de este siglo XXI (Riechmann, 2016), la intención de este artículo es ahondar en las raíces de esta paradoja a fin de desentrañar hasta qué punto la ética de hoy está irremisiblemente condenada a la impotencia o conserva la potencialidad de ser un instrumento de transformación social.

En esta exploración se recalcarán fundamentalmente dos elementos que son considerados las causas fundamentales de este «bloqueo» ético: en primer lugar, las características específicas de las que el marco político y filosófico liberal-capitalista han dotado a la ética contemporánea. Y en segundo lugar, las dinámicas sociales dominantes al día de hoy, que están caracterizadas por la impersonalidad y por una merma significativa de la capacidad de actuación y responsabilidad humanas.

La ética contemporánea como ética liberal 
Pese a que el debate en el seno de la ética es intenso, y cuenta con gran variedad de posiciones, está justificado afirmar que existe una corriente claramente dominante (Hunyadi, 2015: 33-6). Ésta tiene como elemento central a los derechos individuales -no por casualidad los derechos humanos son el estandarte del mundo moderno-, que a su vez se sustentan sobre el doble cimiento que conforman el principio de resolución simbólica de los conflictos y el principio de igual respeto (o iguales derechos y libertades). Los dos elementos constituirían una superación del estadio, en el relato liberal clásico asociado al feudalismo, en el que la vía fundamental de resolución de conflictos habría sido la violencia. Además, también vendría a romper la rigidez de las estructuras sociales feudales en las que cualquier posibilidad de movilidad social se encontraba vetada, asociándose a las diferentes posiciones sociales, con derechos y libertades diferenciados y, por tanto, no universales.

Es decir, la ética hegemónica es una ética de corte liberal que hace de la libertad sinónimo de libertad negativa en el sentido que la entendió Berlin (2000: 191) en su famoso texto Dos conceptos de libertad: una ética de principios, individualista y fragmentada. Esta fragmentación y especialización parcelaria de la ética salta poderosamente a la vista en la lista que Hunyadi realiza y que se reproduce aquí como muestra de la profundidad de este proceso (2015: 35):

La tendencia contemporánea a la parcelación de la ética en diferentes ámbitos concretos: bioética, ética médica, ética de la salud, ética de la minusvalía, ética del final de la vida, ética del medio ambiente, ética animal, ética de la investigación, ética de la ciencia, ética de las nanotecnologías, ética de los negocios, ética 
profesional, ética de la familia, ética sexual, ética del funcionario, ética del soldado, ética de la ciudadanía, ética del psicoanálisis, ética del capitalismo, ética del trabajo, ética social, ética económica, ética de la empresa, ética del juego, ética del turismo, ética de la pedagogía, hay incluso una ética de la creación de la moneda, una ética de la defensa del mar y una ética de la hospitalidad en geriátricos $[\ldots]$

A través de esta característica -la fragmentación-es quizá más sencillo percatarse de que esta formación ética está lejos de ser neutral y conlleva importantes repercusiones políticas y sociales. El carácter fundamentalmente restringido de la ética liberal dominante, la condena a reducir su acción a la defensa de derechos en ámbitos previamente parcelados de la realidad. Es así como el ético de la medicina tendrá que reducir su labor a la definición y posterior defensa de derechos de pacientes y médicos en el marco de la actuación médica, viéndose imposibilitado para cuestionar el marco más amplio en que dichas relaciones se producen y se sitúan. O más importante, aun, sin contar con demasiado margen de maniobra para cuestionar el sistema médico que se toma como punto de partida. En ese sentido, con Hunyadi de nuevo, se puede afirmar que esta ética restringida renuncia a cualquier juicio ético del mundo como un todo y tiene, por tanto, como correlato la sanción implícita del curso global de la sociedad y de las transformaciones que éste implica para los modos de vida. Constituye una renuncia explícita a la posibilidad de una ética global.

Una posición de este tipo encuentra su fundamento en el gran hiato entre el mundo privado y el público que el liberalismo inauguró y ha alimentado desde su nacimiento. Desde su perspectiva, la construcción de un marco de tolerancia y 
de neutralidad sociales pasa por desterrar del ámbito público cualquier ética con aspiraciones globales. De este modo, dichas concepciones, que objetan y dotan de sentido al mundo como un todo, quedan relegadas a la intimidad y se convierten en meras preferencias individuales que en cualquier caso deben subyugarse a la legislación pública y al marco del estado liberal. Un ejemplo paradigmático de esta idea sería la Teoría de la Justicia de Rawls (1995).

Es indudable que tanto en el pasado como en la actualidad, las sociedades que han adoptado concepciones éticas globales, habitualmente religiosas, y las han convertido en sinónimo de un marco legislativo rígido que moldee la vida pública e individual han dado como resultado regímenes muy nocivos para la autonomía de sus poblaciones. Sin embargo, lo que en las últimas décadas parece haberse olvidado es que las supuestas tolerancia y libertad del liberalismo nunca han dejado de ser una promesa incumplida. En primer lugar, porque una libertad estrechamente reducida a libertad negativa olvida el hecho de que más allá de la elección personal existe una multitud de coacciones sistémicas que constituyen obstáculos y límites claros a lo posible. Factores educativos, económicos o geográficos son límites no siempre reconocidos por los defensores de la libertad negativa.

Es en ese sentido en el que Berlan (2016) critica la reducción de la libertad a mera liberación, concepto asociado con matices a la libertad negativa entendida como no intrusión de elementos externos al propio individuo en su toma de decisiones y desarrollo vital. Liberación como emancipación de imperativos comunitarios o religiosos, por ejemplo. Desde su punto de vista, una noción satisfactoria de libertad tiene que nacer como combinación equilibrada de dicha liberación con la autonomía, entendida ésta como una forma de auto- 
gestión que supone un mínimo de independencia material, de control directo sobre las condiciones de vida y de trabajo. Salta a la vista que la autonomía entendida de este modo no es compatible con una ética restringida que en su acción «parcelaria» rechace abordar la cuestión de las condiciones de vida y de trabajo, es decir, de los modos de vida.

También la idea de tolerancia, que el liberalismo propone, suele olvidar que la condición de posibilidad de la misma es precisamente la imposición incuestionada y unilateral de un determinado mundo. Tal y como Marcuse exploró en su texto La tolerancia represiva (2010), a grandes rasgos, la supuesta tolerancia del liberalismo encuentra sus límites precisamente en los discursos y prácticas que ponen en cuestión y pretenden subvertir el orden liberal mismo. Así pues, en la necesidad de ignorar o reprimir dichas estancias reside el carácter represivo de dicho orden. Algunos autores han llegado, incluso, a bautizar como demofascistas a las formaciones liberales contemporáneas en su denuncia de lo que de autoritario y represivo existe en nuestros Estados de Bienestar (García Olivo y Carrión Castro, 2014). En cualquier caso, y lo más relevante para la cuestión que tratamos, es que es innegable que esta tolerancia liberal se convierte en sinónimo de un no cuestionamiento del avance del mundo y de la transformación de los modos de vida que conlleva.

Resulta pertinente terminar recuperando las reflexiones que dedicó a esta idea el filósofo greco-francés Cornelius Castoriadis. Dentro de sus diversos focos de interés, Castoriadis fue un gran crítico del renacimiento de la ética que atestiguó el mundo de las últimas décadas del siglo XX. En su análisis, los siglos XIX y XX habrían sido por derecho propio los «siglos de la política»: 
Napoleón recogido por Hegel: en los tiempos modernos la política ocupa el lugar del fatum antiguo. La era abierta por las revoluciones americana y francesa (y previamente por la Ilustración), continuada durante el siglo XIX por el movimiento democrático y el movimiento obrero [...] es la era que, más que cualquier otro período de la historia humana, parece haber confiado a la política un papel central en la solución de los problemas humanos, convocando a la vez, para bien y a veces para mal, una participación masiva de los pueblos en las actividades políticas (Castoriadis, 1994: 3).

En ese contexto, y en realidad como parte de un proceso que se retrotrae hasta la decadencia de las polis griegas, la ética quedó relegada a disciplina teórica a la que se le dedicaba escaso interés y de la que no se pensaba que tuviera un papel relevante en la mejora del mundo y en la resolución de los conflictos dentro del mismo.

Sin embargo, en las últimas décadas del siglo XX se dieron cita varios episodios que marcarían el renacimiento de la ética en los términos anteriormente descritos. El derrumbe de los regímenes comunistas y su descrédito generalizado, asociado a una progresiva desconfianza en las promesas de emancipación y bienestar a través de los desarrollos técnicos -su nocividad comenzaba a ser innegable en la forma de contaminación, crisis ecológica y desastres industriales y nucleares-, dio pie a un clima de desconfianza generalizada frente a las supuestas capacidades ilimitadas de la política. Así, azuzada por la crisis general de la filosofía y los valores que se vivió en aquellos años, comenzó a surgir con fuerza una ética individual que rechazó la posibilidad de derivar criterios generales para orientar la acción y focalizó sus es- 
fuerzos en la reflexión sobre los actos y los comportamientos singulares. A esta forma de ética, que para Castoriadis se separa ilegítimamente y con nefastos resultados de la política, la denominó ética como encubrimiento.

En su artículo Miseria de la ética, Castoriadis (1994) exploró además los orígenes de esta separación trazando una genealogía que lo llevó hasta Aristóteles y la filosofía helenista, especialmente la estoica. Ante el derrumbe de la forma polis, estos filósofos comenzaron a desarrollar un pensamiento ético en el que las virtudes cívicas, piedra angular de todas las reflexiones anteriores a la suya, pasaban a un segundo plano. La impotencia política les llevó a dar prioridad a transformaciones individuales que pudieran conducir a una vida buena tomando como base en una serie determinada de principios. En el estoicismo se asentará esta idea de que la vida elevada no está ligada a la vida pública, sino más bien al respeto de ciertos principios en la vida privada. Este hiato pasará al cristianismo donde se profundizará y extenderá a muchos lugares del mundo, teniendo su eco en las grandes tradiciones éticas modernas. Tan sólo basta pensar en el filósofo de los principios por excelencia: Kant.

Para concluir, se puede afirmar que la ética contemporánea, en su incapacidad para cuestionar la deriva del mundo y de los modos de vida, actúa más bien avalando el sistema en su conjunto, instalándose como una ética como encubrimiento. Hunyadi habla más bien de un blanqueamiento ético del mundo, producto de una ética que legitima, a través de su quehacer restringido y fragmentario, el grueso del funcionamiento social. Algunos ejemplos relevantes de este blanqueamiento son la impotencia de la bioética frente al galopante proceso de artificialización del mundo, la incapacidad de los comités de evaluación tecnológica para frenar 
el desarrollo tecnológico ${ }^{1}$, la complicidad de muchos comités laborales con la explotación en el trabajo, o el fracaso absoluto que los comités de ética ecológica han tenido a la hora de tratar de frenar una crisis ecológica que no deja de agravarse.

El papel de las dinámicas sociales impersonales

Se abordará ahora la segunda fuente de la paradoja ética de nuestro tiempo. Un concepto fundamental para poder comprenderla correctamente es el de modo de vida de Hunyadi (2015: 47):

El modo de vida se sitúa pues en la interfaz del sistema y de la experiencia social tal como es vivida por los propios actores. Los modos de vida designan así las expectativas de comportamiento impuestas de forma duradera por el sistema a los individuos y a los grupos, y que se imponen independientemente de la voluntad de los actores.

Atendiendo a la definición vemos que el modo de vida, tal y como se ha venido utilizando, tiene particularidades que lo separan de algunos otros conceptos sociológicos similares como práctica social, normal social o habitus. Cuando hablamos de modo de vida el punto de partida es que los comportamientos individuales no vienen determinados por las dinámicas sociales sistémicas. Éstas más bien conforman los modos de vida que en su posición fronteriza entre individuo

1 Decía Langdon Winner en La ballena y el reactor: «Después de que la excavadora nos ha aplastado, podemos levantarnos y medir con cuidado las huellas de las cadenas sobre nosotros. Tal es la impotente misión de la evaluación del «impacto» tecnológico» (1987: 44). 
y sociedad construyen expectativas de comportamiento.

Es en ese sentido en el que se puede hablar de dinámicas impersonales. En su marco de actuación, una serie de principios y fuerzas sociales, asumidos y perpetuados por individuos e instituciones, se imponen socialmente constituyendo un entramado sistémico. Para Hunyadi la lógica en la que los modos de vida se construyen e imponen es una lógica de la emergencia. Lejos de existir una causalidad o una intencionalidad, lo que sucede es que la multitud de acciones aisladas terminan constituyendo a través de sus efectos acumulativos y convergentes una imposición que nadie ha conceptualizado o deseado como tal y en su totalidad. Es decir, lejos de existir un organismo central o poder supremo que diseñe e imponga los distintos modos de vida, éstos son el resultado de un proceso profundamente impersonal en el que el papel que juegan los actores conscientes es mínimo.

En cualquier caso sería un error deducir que en el marco de los modos de vida no tiene cabida ningún comportamiento individual original o toma de decisiones consciente. El hecho de que éstos se impongan en una dinámica impersonal no impide que cualquier individuo pueda crear modos de vida propios y vivir acorde a los mismos. En principio, y eso es quizá la peculiaridad de los marcos capitalistas liberales contemporáneos, no existen prohibiciones explícitas que imposibiliten la construcción de modos de vida diversos. Sin embargo, las limitaciones de una estrategia de este tipo frente a la gran paradoja que se aborda en este artículo son evidentes.

Por un lado, mediante la subversión exclusivamente individual del orden sistémico es prácticamente imposible modificar las dinámicas impersonales hegemónicas, ya que continuarán siendo dominantes y ejerciendo su influjo en el grueso de la población. Por otro lado, partiendo de que en modos de 
vida hegemónicos, las expectativas de vida que dibujan son las mayoritariamente compartidas, el hecho de renunciar explícitamente a los mismos nos pone en un riesgo notable de desocialización y exclusión social (Fraser, 2007). Sin olvidar que los mecanismos de opresión económica y legal hacen casi imposible la reproducción social al margen de instancias como el mercado o el Estado.

De este modo, si seguimos a Hunyadi en sus conclusiones, ninguna acción individual sería capaz de modificar los modos de vida. De esta conclusión se deriva la segunda vertiente de la paradoja ética de nuestro tiempo. Partiendo de que los grandes problemas a los que las sociedades contemporáneas se enfrentan, listados al inicio del artículo, tienen como fuente, precisamente, los modos de vida en el interior de las mismas -consumo, transporte, producción, valores sociales, expectativas, etc.- la conclusión de que la dinámica de los mismos es impersonal y se escapa a cualquier tipo de responsabilidad individual parece dibujar el horizonte de una impotencia ética radical. ¿Cómo abordar, entonces, ese juicio ético global del mundo que diagnosticábamos como imprescindible?

Para salir de este atolladero habría que tomar en consideración una serie de elementos. En primer lugar, Hunyadi introduce un matiz interesante a su posición, considera que existe espacio para acciones colectivas con posibilidades de modificación de los modos de vida. De hecho, como después se verá, su propuesta pasa por la construcción de un órgano institucional que pueda modificar los modos de vida en representación del común. Un ejemplo claro es el neoliberalismo y su enorme capacidad para modificar modos de vida pese a que para muchos su origen se encuentre en una conspiración ideológica exitosa de un grupo relativamente reducido de per- 
sonas (Laval y Dardot, 2013).

Por otro lado, sería interesante tomar en consideración otras reflexiones que han caracterizado la dinámica social impersonal de nuestras sociedades desde otros puntos de vista. Aunque son varios los enfoques que se podrían abordar (Anders, 2011a, 2011b; Berlan, 2012; Ellul, 1980; Jappe, 2016; Jappe, Kurz \& Ortlieb, 2014; Mumford, 2011; Orwell, 2006), me interesa centrarme en lo que Castoriadis planteó al respecto.

A lo largo de toda su obra, pero especialmente en La institución imaginaria de la sociedad (Castoriadis, 1983, 1989), Castoriadis se esforzó por tratar de desentrañar las sutilezas de la relación entre individuos y sociedades. La motivación de su reflexión fue fundamentalmente su deseo de transformar en clave emancipadora a la sociedad en la que le tocó vivir, aunque en sus elaboraciones diera con claves útiles para pensar tanto en sociedades pasadas como en posibles configuraciones sociales futuras.

Resumidamente, y en los términos que ya se analizaron en el artículo El ecomodernismo y su noción de Antropoceno. Un análisis crítico desde la obra de Castoriadis (Almazán Gómez, 2017), el equivalente de los modos de vida en el análisis castoriadiano serían las diferentes significaciones imaginarias sociales que, al entrelazarse de manera coherente, constituyen un determinado mundo instituido. Estas significaciones actuarían de igual modo como expectativas normativas de comportamiento social que constituirían un vector de heteronomía doble: en la vida psíquica de cada individuo y en el comportamiento social del conjunto de la población. En este marco de análisis, las instituciones serían coagulaciones y materializaciones de estas significaciones que estarían caracterizadas por el tipo de dinámica impersonal que antes 
describíamos y que, por tanto, se verían aquejadas de un cierto nivel de automatismo (Almazán Gómez, 2017: 67).

La diferencia fundamental con el planteamiento de Hunyadi es que en la reflexión castoriadiana, la imaginación y la labor creativa están en primer plano. Pese a que las instituciones puedan entrar en dinámicas de inercia autorreproductiva, constituyendo así un vector de heteronomía social, su naturaleza más profunda es imaginaria. De modo que el reto de la emancipación y la transformación social reside precisamente:

[...] en qué medida la sociedad puede reconocer verdaderamente su autocreación en su institución, reconocerse como instituyente, autoinstituirse explícitamente y superar la autoperpetuación de lo instituido y mostrarse capaz de retomarlo y de transformarlo de acuerdo con sus exigencias propias y no de acuerdo con la inercia de aquél, de reconocerse como fuente de su propia alteridad (Castoriadis, 1989: 86).

La raíz de este mundo instituyente que pugna por desbancar al mundo instituido es precisamente la imaginación radical del mundo individual, de donde surgen nuevas significaciones que posteriormente pueden hegemonizarse. De este modo, aunque Castoriadis (1989: 26) parte de una irreductibilidad radical de lo social a lo individual, y en ese sentido reconoce una entidad propia a su equivalente de los modos de vida, establece también mecanismos de conexión entre las dos esferas que mitigan o disuelven la segunda paradoja que en Hunyadi habíamos situado como fuente de una impotencia ética radical.

Conviene, en cualquier caso, no olvidar que las trans- 
formaciones sufridas por nuestro mundo en las últimas décadas, sobre todo en lo referente al desarrollo tecnológico, dibujan nuevos obstáculos para la posibilidad de una acción ética. En concreto, Günther Anders (2011a) insistió numerosas ocasiones en que nuestro hoy era el escenario de un desnivel prometeico. En esta fórmula resumía la circunstancia inédita en la historia de la humanidad de un entramado sistémico y unos instrumentos tan poderosos que habían sido capaces de generar un hiato entre la capacidad de responsabilidad individual humana y su propia potencia transformativa. En su ejemplo paradigmático, ¿cómo asimilar la responsabilidad moral derivada de pulsar el botón que lanzara una bomba nuclear que pudiera acabar con cientos de miles de vidas? Tal y como ya exploré en un artículo breve (Almazán Gómez, 2016), esta condición de inasimilabilidad se ha extendido hasta las acciones más cotidianas de nuestro día a día, obligándonos en primer lugar a asumir el reto de conseguir hacernos responsables de nuestras acciones, lo que pasa en primer lugar por hacernos conscientes de sus repercusiones en el mundo hiperconectado y delirante donde nos ha tocado habitar.

\section{Conclusión}

Haciendo un balance de lo expuesto se pueden dibujar algunas vías posibles para desenredar el nudo que constituye la gran paradoja ética de nuestro tiempo en los términos en que la hemos definido.

La primera acción insoslayable es la ruptura del consenso liberal. Cualquier solución debe necesariamente pasar por abandonar la concepción dominante de ética restringida o, en los términos de Hunyadi, pequeña ética, para dar paso a una gran ética capaz de una evaluación de los modos de 
vida. En sus trabajos, Riechmann (2005: 59) ha realizado una identificación explícita entre esta transición y la reapertura al mundo político. Frente a las retóricas del fin de la historia y una ética liberal atrapada en los principios y el refinamiento del ámbito individual, el autor madrileño nos invita a revertir la separación de ética y política que se encuentra en la raíz de la institución contemporánea de la ética como encubrimiento. Así, muy en sintonía con Hunyadi, Riechmann propone entender que ambos están intrínsecamente unidos y no son separables hablando de la ética como microética y de la política como macroética.

Por otro lado, frente a las dinámicas impersonales, el objetivo sería desarrollar estrategias y formas políticas que permitan tomar decisiones colectivas, directas y democráticas sobre el desarrollo material e institucional del mundo. La consecuencia sería un control análogo sobre la naturaleza y transformación de los modos de vida. Desde aquí puede surgir una gran variedad de posiciones y propuestas, que variarán dependiendo del tipo de análisis de las dinámicas impersonales que realicemos. Por ejemplo, la propuesta de Hunyadi (2015: 87) es la creación de un parlamento virtual de los modos de vida. Éste sería una institución europea en la que, a través de internet, toda la población podría participar en la toma de decisiones vinculantes y democráticas sobre los modos de vida. Esta postura parte de una dudosa identificación entre lo común y lo estatal y de una confianza en las posibilidades de la política institucional como acción consciente para, efectivamente, modificar el rumbo de las transformaciones en los modos de vida. Existen, por otro lado, las posiciones que defienden una reformulación y radicalización democrática de instancias como los comités de evaluación tecnológica (Linares, 2008). Aquí se realiza una diferenciación relevante 
entre el estado y la sociedad civil, que se vería reflejada en los comités. Sin embargo, sigue habiendo una confianza excesiva en el potencial de la acción política en el sentido convencional frente a las dinámicas impersonales.

Si se atiende a la profunda conexión que existe entre los modos de vida y el mundo instituido en el sentido castoriadiano, los límites de estrategias como las anteriores saltan a la vista. Para poder evaluar y transformar los modos de vida sería necesario, por un lado, transformar la colección de significaciones imaginarias sociales dominantes en nuestras sociedades (Erreguerena Albaitero, 2002). Para percatarse del tremendo vuelco que algo así supondría, basta con pensar en que algunas de las significaciones que habría que derrocar son la racionalidad del mercado o la omnipotencia de la técnica (Castoriadis, 2006).

Por otro lado, habría que moverse hacia un nuevo mundo instituido compatible precisamente con la acción consciente y democrática. Para ello, en primer lugar, tal y como se discutió más arriba, sería imperativo acabar con la forma de alienación que supone la relativa autonomización de las instituciones y su reducción del margen de acción consciente. Además, supondría re-escalar el mundo a nivel material de modo que éste recuperara su dimensión humana y se hiciera de nuevo compatible con la posibilidad de reponsabilidad individual y colectiva. Eso o, siguiendo la sugerencia de Anders, enfrascarnos en ejercicios de estiramento moral que nos permitieran hacernos cargo del mundo que hemos construido. Una vez superados dichos escollos, restaría la no menor dificultad de materializar lo que Castoriadis denominó el imaginario autónomo frente al imaginario capitalista. Este tiene como condición el desarrollo de una autonomía individual y social (Castoriadis, 1983: 173-183) que precisamente dibujaría 
un escenario en que tales tipos de decisiones sobre los modos de vida se harían posibles.

En cualquier caso, y para terminar, debemos recordar que, aunque no se abordará a profundidad esta cuestión, la idea de una desalienación total que nos hiciera transparentes para nosotros mismos y además fuera punto de partida de una posibilidad de plasticidad social infinita ha sido ya muy criticada (Kolakowski y Hampshire, 1976). Todo parece indicar que, ya sea en la forma de las dinámicas del subconsciente ${ }^{2}$, los límites materiales y ecológicos del planeta, el mismo carácter limitado de nuestras capacidades como animales humanos o el mínimo de estabilidad e inercia que el mundo institucional humano necesita para ser operativo y un nivel mínimo de alienación en la vida individual y colectiva del ser humano parece ser simplemente inevitable.

\section{Bibliografía}

Almazán Gómez, M. A. (2016, diciembre 7). El reto éticopolítico de hacernos cargo de nuestras acciones [Blog de noticias]. Recuperado a partir de http://www. animalpolitico.com/blogueros-una-vida-examinadareflexiones-bioeticas / 2016/12 / 07 / reto-etico-politicohacernos-cargo-nuestras-acciones /

. (2017). El ecomodernismo y su noción de Antropoceno. Un análisis crítico desde la obra de Castoriadis. Iberoamérica Social: Revista-red de estudios sociales, VII,

2 En el análisis de Castoriadis en cualquier caso el hecho de que existan dinámicas inconscientes no es sinónimo de alienación o heteronomía. Ésta es más bien fruto de la implantación de la idea de otro en nuestra psique y, especialmente, de nuestra repetición de dicha idea arreflexivamente en nuestro comportamiento individual y social. 
61-90.

Anders, G. (2011a). La obsolescencia del hombre (Vol. I). Sobre el alma en la época de la segunda revolución industrial. Valencia: Pre-textos. . (2011b). La obsolescencia del hombre (Vol. II). Sobre la destrucción de la vida en la época de la tercera revolución industrial. Valencia: Pre-textos.

Barba, A. (2016). La risa canibal: humor, pensamiento cínico y poder (Primera edición). Barcelona: Alpha Decay.

Berlan, A. (2016). Autonomie et délivrance. Repenser l'émancipation à l'ère des dominations impersonnelles. Revue du MAUSS, (48), 59-74.

. (2012). La fabrique des derniers hommes: retour sur le présent avec Tönnies, Simmel et Weber. Paris: La Découverte.

Berlin, I. (2000). The Proper Study of Mankind: an Anthology of Essays. (H. Hardy, Ed.) (1. paperback ed). New York: Farrar, Straus and Giroux.

Castoriadis, C. (2006, febrero). Reflexiones sobre el «desarrollo»y la «racionalidad». Recuperado el 28 de septiembre de 2016, a partir de http://www.fundanin.org/ castoriadis7.htm

. (1994). Miseria de la ética. Zona Erógena, 22, 1-14. . (1989). La institución imaginaria de la sociedad. Vol 2. El imaginario social y la institución (Vol. 2). Barcelona: Tusquets.

. (1983). La Institución imaginaria de la sociedad. Vol 1. Marxismo y teoría revolucionaria (Vol. 1). Barcelona: Tusquets.

Ellul, J. (1980). The Technological System. (J. Neugroschel, Trad.). New York: Continuum.

Erreguerena Albaitero, M. J. (2002). Cornelius Castoriadis: sus conceptos. En Anuario de investigación 2001, Vol II (pp. 3947). México, D.F.: UAM-X, CSH, Depto. de Educación y 
Comunicación. Recuperado a partir de http:/ / bidi.xoc. uam.mx/tabla_contenido_libro.php?id_libro=32

Fraser, N. (2007). Identity, Exclusion, and Critique: A Response to Four Critics. European Journal of Political Theory, 6(3), 305-338. https: / / doi.org/10.1177/1474885107077319

García Olivo, P. \& Carrión Castro, J. C. (2014). Dulce Leviatán.

Críticos, víctimas y antagonistas del Estado del Bienestar (Primera). Bardo ediciones.

Hunyadi, M. (2015). La tiranía de los modos de vida. Sobre la paradoja moral de nuestro tiempo. (F. González Fernández, Trad.) (1a). Madrid: Cátedra.

Jappe, A. (2016). Las aventuras de la mercancía. Logroño: Pepitas de Calabaza.

Jappe, A., Kurz, R. \& Ortlieb, C. P. (2014). El absurdo mercado de los hombres sin cualidades: ensayos sobre el fetichismo de la mercancía. Logroño: Pepitas de Calabaza.

Kolakowski, L. \& Hampshire, S. (1976). El mito de la autoidentidad humana. La unidad de la sociedad civil y la sociedad política. (J. Álvarez Valencia, trad.). Valencia: Universidad de Valencia.

Laval, C. \& Dardot, P. (2013). La nueva razón del mundo: ensayo sobre la sociedad neoliberal. Barcelona: Gedisa.

Linares, J. E. (2008). Ética y mundo tecnológico (1 $\left.{ }^{\mathrm{a}}\right)$. México, D.F: UNAM-Facultad de Filosofía y Letras/Fondo de Cultura Económica.

Marcuse, H. (2010). La Tolerancia represiva y otros ensayos. (C. de Vicente Hernando, trad.). Madrid: Los Libros de la Catarata.

Mumford, L. (2011). El pentágono del poder: El mito de la máquina (dos). La Rioja: Pepitas de Calabaza Ed.

Orwell, G. (2006, octubre). La civilización de la máquina 
(Cap. XII de El camino de Wigan Pier). Resquicios, I (2), 10-23.

Rawls, J. (1995). Teoría de la justicia. Distrito Federal: Fondo de Cultura Económica. Recuperado a partir de http: / / public.eblib.com / choice/ publicfullrecord. aspx?p=4508399

Riechmann, J. (2016). El no actuar en aquellos días... Apuntes sobre la crisis ecosocial. Madrid: Foro Transiciones.

(2005). Un mundo vulnerable: ensayos sobre ecología ética y tecnociencia. Madrid: Los Libros de la Catarata.

Winner, L. (1987). La ballena y el reactor: una búsqueda de los limites en la era de la alta tecnología. Barcelona: Gedisa Editorial. 


\section{Resumen}

En este artículo se aborda la que Hunyadi ha definido como la gran paradoja ética de nuestro tiempo. A la que quizá sea la mayor presencia social de la ética en toda la historia parece corresponderle una incapacidad casi total para evaluar y modificar los modos de vida en el interior de nuestras sociedades. Para dar cuenta de este impasse se abordan dos fuentes del mismo. En primer lugar, la matriz liberal de una ética que se establece como ética restringida -en tanto que individual, de principios y fragmentada-. Por otro, la presencia dominante de dinámicas sociales impersonales que dificultan la actuación individual para revertir la situación de automatismo en la producción y el mantenimiento de los modos de vida. El artículo finaliza señalando algunas posibles salidas a esta paradoja.

Palabras clave: modos de vida, Hunyadi, ética, política, Castoriadis.

\section{Abstract}

This article will address what Hunyadi has defined as the greatest ethical paradox of our time. In a moment where ethics is more socially present than ever, we present an almost total inability to evaluate and modify the ways of life within our societies. To account for this impasse, two sources will be addressed. First, the liberal matrix of an ethic that establishes itself as restricted ethics- as individual, principled and fragmented. On the other, the dominant presence of impersonal social dynamics that hinder individual action to reverse the situation of automatism in the produc- 
tion and maintenance of ways of life. The article ends by pointing out some possible outputs to this paradox.

Key words: ways of life, Hunyadi, ethics, politics, Castoriadis. 